\title{
Macroscopic Single-Crystal Gold Microflakes and Their Devices
}

\author{
Jiyu Zhou, Abhijit Saha, Jozef Adamcik, Haiqing Hu, Qingshan Kong, Chaoxu Li, * \\ and Raffaele Mezzenga*
}

Nature offers countless examples of systems with remarkable 2D physical properties, e.g., anisotropic conductivity of graphite, ${ }^{[1]}$ optical properties of nacre, ${ }^{[2]}$ and superstrong mechanical property of bones. ${ }^{[3]}$ These intriguing shape/size-dependent properties have inspired the synthesis of a wide variety of 2D-layered nanomaterials. ${ }^{[4]}$ In particular, the quantum 2D confinement of electrons found in graphene nanosheets implies that 2D metal nanomaterials may bear unique catalytic, electrical, magnetic, and optical properties controlled by their geometric feature. ${ }^{[5]}$ For example, gold platelets/nanosheets have size-dependent surface plasmon absorption in the visible-near-infrared region, and their sharp edges and corners can act as nanofocus sources to improve fluorescence, Raman scattering, single-molecule detection and nonlinear nanospectroscopy. ${ }^{[6]}$ Their large aspect ratio can assist in the top-down fabrication of optical nanocircuitry for high-speed communication, ${ }^{[7]}$ as well as in the organization into layered composites for specific functions. ${ }^{[8,9]}$ The atomically flat surfaces of single-crystal gold 2D nanomaterials provide an ideal platform for single-molecule sensing and imaging. ${ }^{[7]}$ However, it remains highly challenging to transfer this 2D effect to macroscopic length scales serving technological applications in catalysis, sensing, optoelectronic devices and conductive composites.

In the past decades, much endeavor has been devoted to develop shape/size-controlled bottom-up approaches to synthesize gold 2D nanomaterials with high yield. Micro- or sub-micrometric single-crystal gold platelets were frequently prepared by reducing chloroaurate ions with wet chemical, electrochemical, photochemical, and thermal reduction methods in the presence of soft templates, capping agents or interfaces. ${ }^{[10-15]}$ Single-crystal gold nanosheets with the planar area up to $10^{2} \mu \mathrm{m}^{2}$ were also synthesized from chloroaurate ions by several research groups, such as by Jiu et al. in the presence of

J. Zhou, Prof. Q. Kong, Prof. C. Li

Qingdao Institute of Bioenergy and Bioprocess

Technology

Chinese Academy of Sciences

Songling Road 189, Qingdao 266101, P.R. China

E-mail: licx@qibebt.ac.cn

I. Zhou, Prof. H. Hu

Department of Polymer Science and Engineering

Qingdao University of Science and Technology

Zhengzhou Road 53, Qingdao 266042, P.R. China

Dr. A. Saha, Dr. J. Adamcik, Prof. R. Mezzenga

ETH Zurich, Food and Soft Materials

Department of Health Science \& Technology

Schmelzbergstrasse 9, LFO, E23 8092, Zürich, Switzerland

E-mail: raffaele.mezzenga@hest.ethz.ch

DOI: 10.1002/adma.201405121 ethylene glycol and polyvinylpyrolidone, ${ }^{[16]}$ by Qin et al. in the presence of dodecyl glyceryl itaconate, ${ }^{[17]}$ and by Li et al. in the presence of ionic liquid. ${ }^{[18]}$ Recent efforts have converged on environmental friendly and low cost synthesis routes, particularly exploiting the use of biomolecules and biopolymers, e.g., chitosan, ${ }^{[19]}$ arginine, ${ }^{[9]}$ combination of $\mathrm{H}_{2} \mathrm{O}_{2}$ and starch, ${ }^{[10]}$ and amyloid fibrils. ${ }^{[8]}$ In all the above cases, chloroaurate ions were reduced in a controllable way, and gold single crystals underwent a nucleation and 2D growth process from sub-nanometric to micrometric lateral length scales. Yet, and despite several examples of macroscopic crystals found in nature, to the best of our knowledge, these synthesis approaches have not been successful to extend the single-crystal gold nanosheets to macroscopic scales, or, in other words, to a length scale accessible to the human eye resolution regime, i.e., with planar area $\geq 10^{3} \mu \mathrm{m}^{2}$ (microflakes). The only macroscopic gold microflakes reported to date are gold foils engineered from nugget in top-down approaches, e.g., via vapor deposition and hammering, which, however, are incredibly energy-consuming, polycrystalline and with rough surfaces. ${ }^{[20-22]}$ Thus, a simple, green and efficient method to prepare macroscopic single-crystal gold microflakes remains challenging, yet highly desirable in synthetic chemistry, material science, and nanotechnology.

In sharp contrast to the previous techniques of directionally growing 2D gold crystals using templates and interfaces, ${ }^{[17,23,24]}$ it was recently found that certain biomaterials or their assemblies alone could act as both reducing agent and capping agent to prepare $10^{2} \mathrm{~mm}^{2}$ single-crystal gold nanosheets..$^{\left[{ }^{[8]}\right.}$ Herein, we show that by carefully tuning the concentrations of chloroaurate ions and amyloid fibrils as well as their ratio, singlecrystal gold microflakes with an unprecedented planar area over $10^{4} \mathrm{\mu m}^{2}$ and thickness larger than $100 \mathrm{~nm}$ can be prepared in a simple and highly scalable one-step green synthesis process. Despite being two orders of magnitude larger than the 2D gold single crystals previously reported in literature, they remain single-crystalline with regular shapes and atomically flat surfaces. Production of large gold microplates with thickness exceeding $100 \mathrm{~nm}$ has immediate relevance in a number of fields. For example, edge sizes exceeding $100 \mathrm{~nm}$ allow edible and cosmetic applications without the compulsory declaration of use of nanoparticles in the formulation, in agreement with European regulations (ISO/TS 27687:2008). Moreover, we demonstrate that these new materials enable producing flexible and electrically conductive composite films with strain/ force-sensing properties in a facile, one-step casting method.

The synthesis of single-crystal gold microflakes was carried out at $60{ }^{\circ} \mathrm{C}$ and $\mathrm{pH} 2$ in aqueous solutions containing predetermined concentrations of hydrogen tetrachloroaurate and $\beta$-lactoglobulin amyloid fibrils. Amyloid fibrils are 


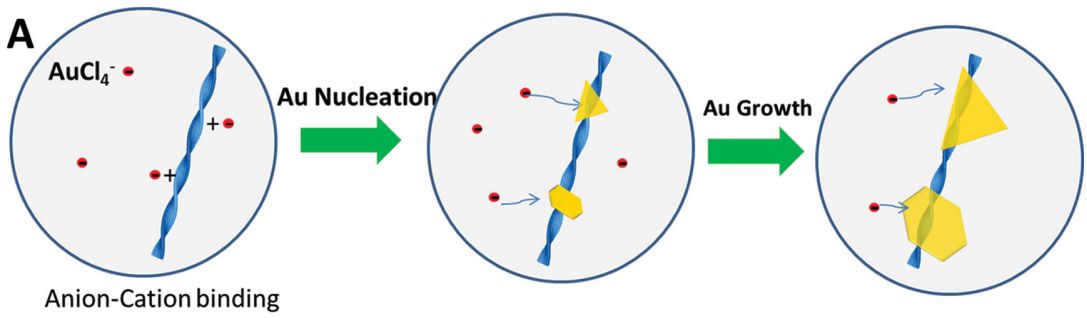

Anion-Cation binding

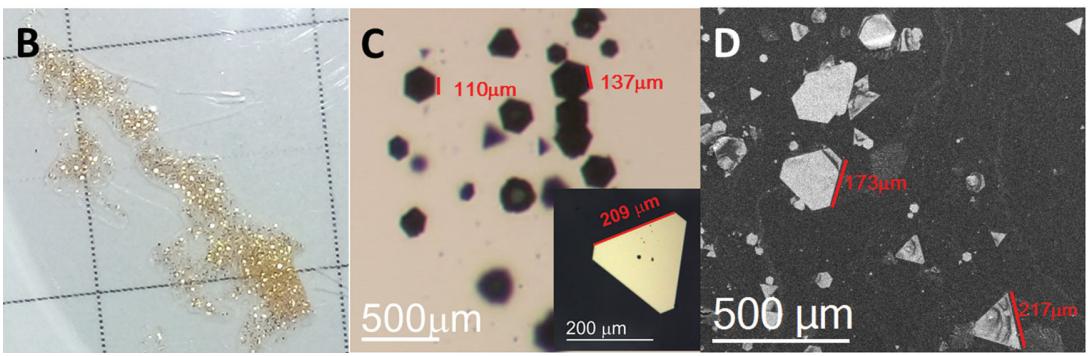

Figure 1. A) Schematic synthesis procedure of macroscopic single-crystal gold microflakes from chloroaurate ions and amyloid fibrils, and their images by B) visual observation (grid size $\left.0.9 \times 0.9 \mathrm{~cm}^{2}\right)$; C) optical microscopy; and D) SEM

protein-based filaments organized laterally and twisted into nanofibrils with the diameter of $1-6 \mathrm{~nm}$ and the persistent length over $1.0 \mu \mathrm{m}$, whose multiple functions as reducing, directing, and stabilizing agents in synthesizing gold platelets have been proposed in our previous research. ${ }^{[8,25,26]}$ $\beta$-lactoglobulin for which fibrillization procedure is well-established (e.g., protein $2 \mathrm{wt} \%$, ionic strength $<1 \times 10^{-3} \mathrm{M}, \mathrm{pH} 2$, $90{ }^{\circ} \mathrm{C}$, and $\left.>5 \mathrm{~h}\right),{ }^{[26-28]}$ was selected as a model protein due to its combination of low cost, food grade, and broad availability. As schemed in Figure 1A, chloroaurate ions partially bind the surfaces of positively charged amyloid fibrils after mixing homogenously, and are subsequently reduced to elemental gold and forming nuclei. Free available chloroaurate ions continuously diffusing from the solution continue reducing around the nuclei, eventually yielding growing single-crystal gold platelets with final size depending on the delicate balance between nucleation and growth kinetics. After carefully optimizing the experimental conditions, single-crystal gold microflakes can be obtained with their lateral edge size over $0.1 \mathrm{~mm}$. In this study, we classify the 2D gold materials into three categories according to their planar area: platelets $\left(<1 \mu \mathrm{m}^{2}\right)$, nanosheets $\left(1-10^{3} \mu \mathrm{m}^{2}\right)$, and microflakes $\left(>10^{3}{\mu \mathrm{m}^{2}}^{2}\right.$ (Figure $\mathrm{S} 1$, Supporting Information).

Figure 1B-D shows representative images of the resulting $2 \mathrm{D}$ gold materials with microflakes, where amyloid fibrils are invisible due to their nanoscale diameter (Figure S2, Supporting Information). Due to their large planar area over $10^{4}{\mu \mathrm{m}^{2}}^{2}$ and atomically flat surfaces, individual gold microflakes can be easily distinguished by naked eyes in Figure 1B. Brightness, darkness, and golden color arise due to different reflecting angles of microflakes to the camera lens. Further observations via optical microscopy (OM) and scanning electron microscopy (SEM) in Figure 1C,D, reveal hexagonal and triangular geometries in a symmetry characteristic of their (111) Fm3m plane and single crystalline features. ${ }^{[8,25]}$ With the coexistence of platelets and nanosheets, a few microflakes have the lateral dimension up to $217 \mu \mathrm{m}$ in the case of triangle, and $137 \mu \mathrm{m}$ in the case of hexagon, cor-

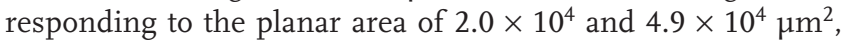
respectively. The lateral edge size is one order of magnitude larger than that of single-crystal gold nanosheets we synthesized before; whereas, the planar area is two order of magnitude larger (for a comparison of the triangular geometry see Figure S3, Supporting Information). ${ }^{[8]}$

The synthesis conditions of microflakes were optimized by varying concentrations of chloroaurate ions and amyloid fibrils under constant $\mathrm{pH} \approx 2$ and temperature $\approx 60^{\circ} \mathrm{C}$. As shown in Figure 2 , by adding $0.5,0.8$, and $1.5 \times 10^{-3} \mathrm{M}$ chloroaurate ions into $0.017 \pm 0.001 \mathrm{wt} \%$ amyloid fibrils solution (corresponding to Point A, B, and C in Figure 3), the planar area of the largest
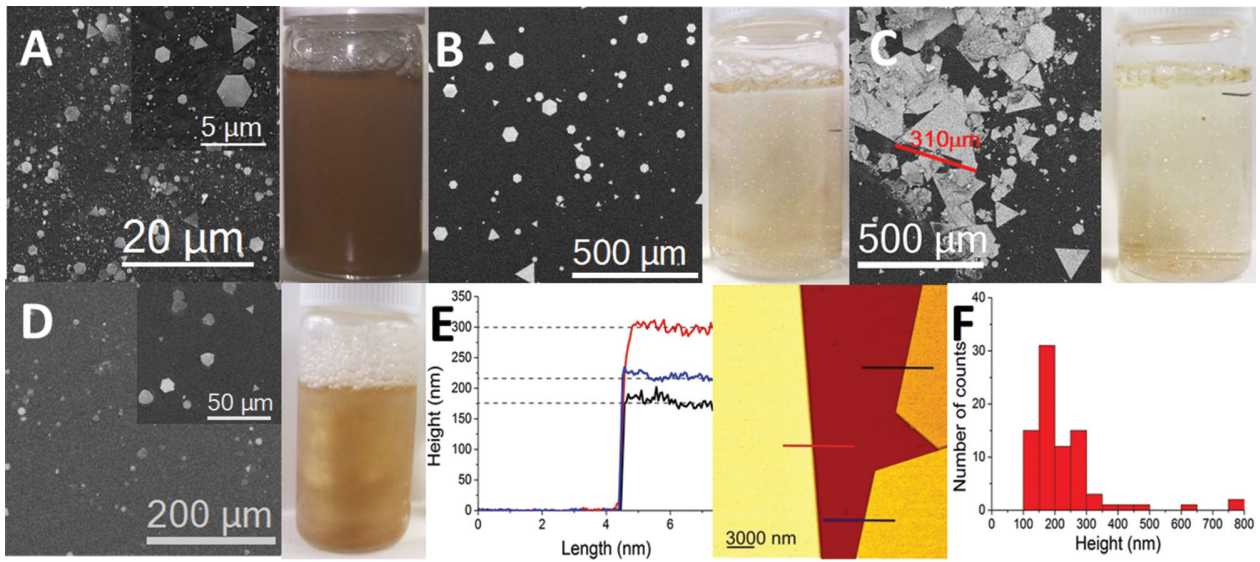

Figure 2. A-D) SEM images (left side) and visual observation (right) of single-crystal 2D gold materials with increasing planar area synthesized with chloroaurate ions and amyloid fibrils of A) $0.5 \times 10^{-3} \mathrm{M}, 0.017 \mathrm{wt} \%$; B) $0.8 \times 10^{-3} \mathrm{M}, 0.017 \mathrm{wt} \%$; C) $1.5 \times 10^{-3} \mathrm{M}, 0.017 \mathrm{wt} \%$; D) $3.2 \times 10^{-3} \mathrm{M}, 0.67 \mathrm{wt} \%$. E) Height profiles (left) along the indicated lines in the AFM image (right) of microflakes. F) Thickness histogram plot of single-crystal 2D gold materials. 


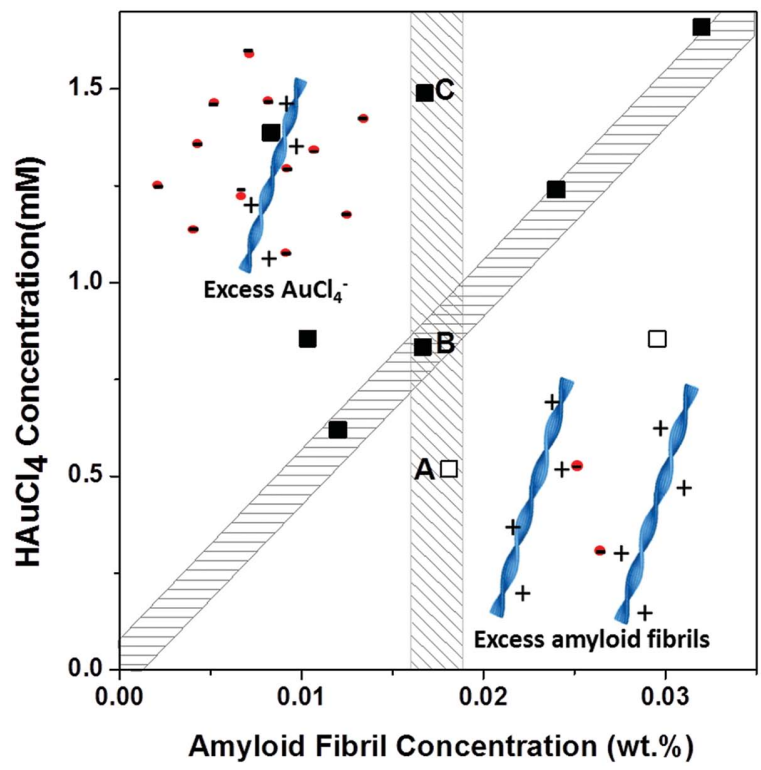

Figure 3. Concentration variation of amyloid fibrils and chloroaurate ions for synthesis of single-crystal $2 \mathrm{D}$ gold materials at $\mathrm{pH} \approx 2$ and $60^{\circ} \mathrm{C}$ : microflakes (closed symbols) and platelets/nanosheets (open symbols). The insets illustrate two regions separated by a dotted line where there are excess chloroaurate ions or excess amyloid fibrils.

2D gold objects increases 1 to 2 orders of magnitude from $6.5 \times 10^{1}$ (Figure 2A), $3.5 \times 10^{3}$ (Figure 2B) to $8.3 \times 10^{4} \mu^{2}$ (in Figure 2 C). Consistently, the predominant presence of platelets of $\approx 10^{0} \mu \mathrm{m}^{2}$ in Figure 2A yields the characteristic brownish color of the solution. The existence of macroscopic microflakes can be confirmed by visual observations in Figure 2B,C, where mirror-like surfaces of microflakes are distinguishable by naked eyes. Notably, increasing the fibril concentration by over $0.1 \mathrm{wt} \%$ is deleterious to microflakes synthesis, e.g., no microflake was observed by varying the concentration ratio between chloroaurate ions and amyloid fibrils (see Figure 2D). ${ }^{[8]}$ This implies that the presence of amyloid fibrils promoting nucleation needs to be maintained low in order to favor growth over nucleation of the crystals.

Atomic force microscopy in Figure 2E further reveals thickness variation of these microflakes, measured by the height profiles along indicated lines. Complete microflakes were not shown due to their large dimensions over thickness. In contrast to $\approx 25 \mathrm{~nm}$ thickness measured for nanosheets (Figure S4, Supporting Information), all these microflakes have thickness above $100 \mathrm{~nm}$, indicating that their unprecedented lateral dimension is occurring together with an average increase in their thickness. The thickness can go as far as $\approx 800 \mathrm{~nm}$ (Figure $2 \mathrm{~F}$ ), eight times larger than the nanosheet thickness of $100 \mathrm{~nm}$ ever synthesized through an interface-template method. ${ }^{[17]}$

The conditions to synthesize single-crystal gold microflakes are summarized in Figure 3, where both the concentrations of chloroaurate ions and amyloid fibrils were varied. It was found that a hypothetical boundary (indicated as the black dotted line), separated two regions for 2D gold nanomaterials: microflakes and nanosheets/platelets. This promptly highlights the manifold roles of amyloid fibrils for gold synthesis not only as the reducing, directing, and stabilizing agents, ${ }^{[8,25]}$ but also as the nucleation agent. At higher concentrations of amyloid fibrils, chloroaurate ions reduce faster and concentration of nuclei increase, therefore favoring the formation of 2D nanomaterials with smaller size: nanosheets/platelets. On the contrary, higher concentrations of chloroaurate ions favor the growth process of gold, therefore resulting in the formation of 2D nanomaterials with larger size: microflakes.

The increase in size to macroscopic length scale opens up new possibilities for the manufacturing across scales of these materials. For example, microflakes have a stronger tendency to deposit horizontally on substrates when sedimentation occurs naturally in suspensions, compared with gold nanoplatelets, ${ }^{[8]}$ nanocomposites of amyloids and grapheme, ${ }^{[1]}$ and clay-based nanocomposite hydrogels, ${ }^{[29]}$ for which, 2D layerby-layer orientation is more efficiently achieved by vacuumfiltration methods. Thus, stretchable, conductive composites can be designed by a simple one-step casting approach. For example, the gold microflakes (indicated in Figure 2B) dispersed in a water-dispersion of polyurethane and then casted on polystyrene substrates, leading to free-standing composite films after completely air-drying. As shown in Figure 4A, even with less than $10 \mathrm{wt} \%$ gold, the film shows homogenous metallic gold color, with smooth and shiny surfaces. Color variation from golden to black, as identified by optical microscopy in Figure 4B, confirms the mirror-like reflecting surfaces of single-crystal microflakes. Further SEM observation of their cross-sections in Figure 4C suggests that gold 2D nanomaterials concentrated and organized layer-by-layer on the bottom sides of the films, as a consequence of their high density $\left(19.32 \mathrm{~g} \mathrm{~cm}^{-3}\right.$ for pure gold in contrast to $\approx 1.2 \mathrm{~g} \mathrm{~cm}^{-3}$ for polyurethane and $1.0 \mathrm{~g} \mathrm{~cm}^{-3}$ for water). For the sake of comparison, nanosheets (indicated in Figure 2D) were also used to prepare control films (shown in Figure S5A, Supporting Information). In sharp contrast to the insulating composite film containing nanosheets, the film with microflakes is highly conductive on the bottom side (Figure S5B, Supporting Information), withinplane conductivity as high as $4.9 \times 10^{3} \mathrm{~S} \mathrm{~cm}^{-1}$, i.e., two orders of magnitude lower than that of pure gold $\left(4.1 \times 10^{5} \mathrm{~S} \mathrm{~cm}^{-1}\right)$. This indicates that increasing the $2 \mathrm{D}$ nanomaterials' size from nanosheets to microflakes leads to sedimentation-driven denser packing, with percolating flakes over the casted film. Moreover, the in-plane conductivity remains constant when bending, twisting or rolling the film (Figure S6, Supporting Information), making the film an ideal candidate for flexible conductors in applications as diverse as flexible displays, field-effect transistors, actuators, electrodes, and smart clothing, etc.

The stretching experiment shows that the films can attain a strain over $500 \%$ without failure (Figure S7, Supporting Information). After the strains of $28 \%$ (State $\alpha$ ), $45 \%$ (State $\beta$ ), and $55 \%$ (State $\gamma$ ), the stretched films were released and labeled as Sample $\alpha, \beta$, and $\gamma$, respectively, as shown in Figure 4D. During stretching, Sample $\alpha$ remained highly conductive up to $17.5 \%$ and became insulating at a strain of $27 \%$ (Figure 4E). This strain-controlling switch effect has been already reported in elastomers' blending or coating with zero/1D metal fillers, ${ }^{[30-34]}$ and with nanosheets coating or patterning flexible films. ${ }^{[9]}$ 

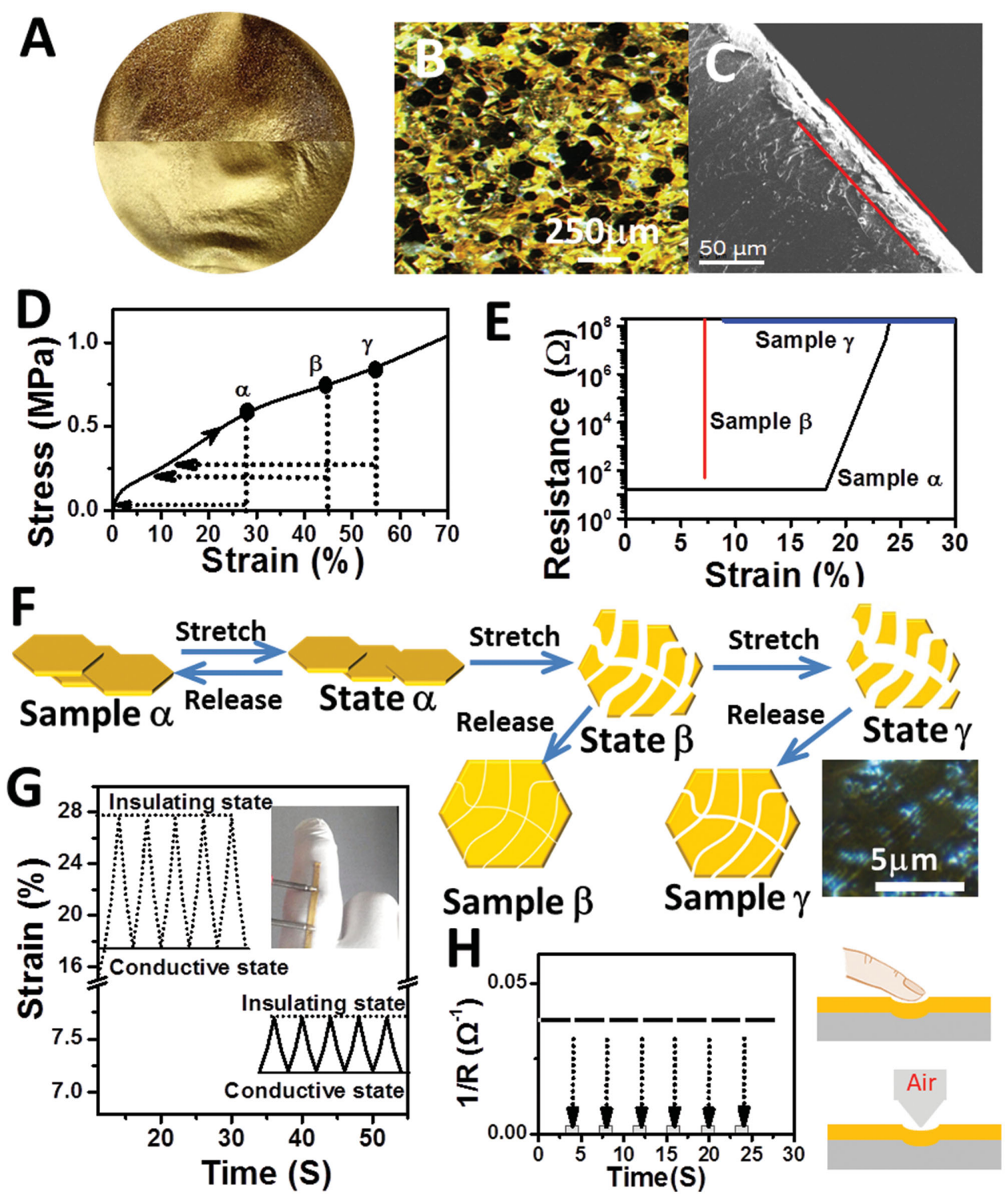

Figure 4. A-C) Front (upper part) and back (bottom part) view of the free-standing polyurethane films (thickness $100 \mu \mathrm{m}$ ) with microflakes as observed by visual observation (A), optical microscopy (B), and SEM cross-section images (C). The gold nanoflake layer is indicated by red lines. D) Stress-strain curve when unaxially stretching the film. Three strains of $27.5 \%(\alpha), 45 \%(\beta)$, and $55 \%(\gamma)$ were reached (labeling as State $\alpha, \beta$, and $\gamma$, respectively) and subsequently released (labeling as Sample $\alpha, \beta$. and $\gamma$, respectively). E) Strain-dependence of in-plane electric resistance (R) of Samples $\alpha, \beta$, and $\gamma\left(\right.$ strip size $5 \times 10 \mathrm{~mm}^{2}$ ). F) Schematic representation of microflakes in the film at State $\alpha, \beta$, and $\gamma$ and Sample $\alpha, \beta$, and $\gamma$. The inset gives an optical microscopy image of a nanoflake in State $\gamma$. G) Strain-controlled in-plane conductive and insulating states for Sample $\alpha$ (dotted line) and $\beta$ (solid line), which can be utilized for sensing finger bending (the inset image). H) Variation of in-plane electric resistance of the film under finger touch ( $0.037 \mathrm{~N}$ ) and air force $(0.02 \mathrm{~N})$ from a nozzle with the diameter $0.5 \mathrm{~mm}$.

Surprisingly, Sample $\beta$ has a conductivity switch effect very sensitive to its strain variation, which, to our knowledge, has not been observed before in flexible conductive materials with zero/1D metal fillers. It shifted from metallic conductivity to insulation within a strain variation of $0.5 \%$ (Figure $4 \mathrm{E}$ ). This behavior resulted from breaking the microflakes in the polyurethane matrix when the film is stretched. As shown in Figure $4 \mathrm{~F}$, the strain variation first separated the microflakes and then broke the microflakes into pieces (see the inset images in Figures 4F, and S8, Supporting Information). Once the strain was higher than 55\%, the gold pieces were not capable of recovering their initial places; therefore giving a strain-independent insulation state (Sample $\gamma$ in Figure 4E).

The alteration of in-plane conductive behavior through simply stretching the film to various strains offers an ideal platform for diverse applications, such as strain-sensing devices and electric controllers. For example, from Sample $\alpha-\beta$, the increase in strain-sensitivity offers the flexibility of designing an "on-off" switch controlled by different bending degrees in robotics applications (Figure $4 \mathrm{G}$ ). The high strain-sensitivity of 

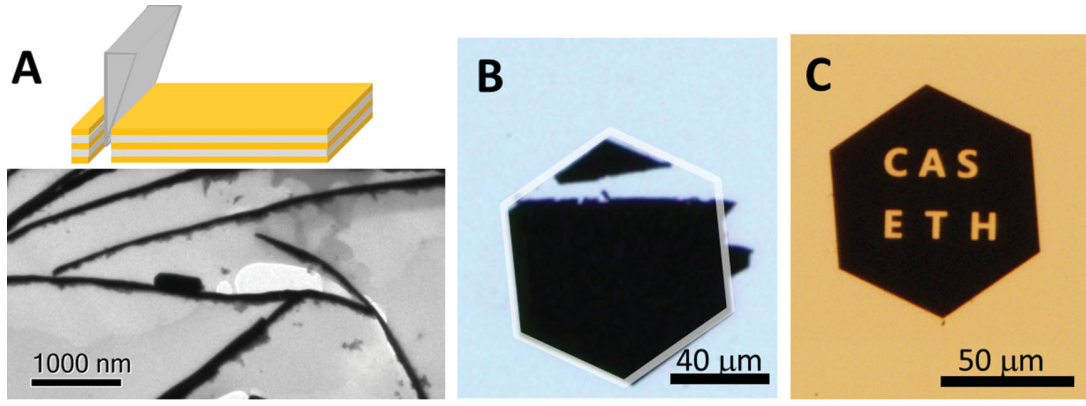

Figure 5. A) Nanoskiving layer-by-layer organized microflakes for preparation of gold singlecrystal nanowires imaged by TEM. B) Removal of one corner from a gold single-crystalline hexagon by mechanical cutting, as imaged by optic microscopy. C) Focused ion beam etching a gold single-crystal microflake with specific letters.

Sample $\beta$ can further be used to sense force as low as $0.02 \mathrm{~N}$ (Figure $4 \mathrm{H}$ ). For instance, a finger touching the film or an air flux (pressure of $0.1 \mathrm{~N} \mathrm{~mm}^{-2}$ ) blowing on it could alter the inplane electric properties of the film from conductive to insulation states. Similarly, this behavior was also used to sense weak vibration (Figure S9, Supporting Information).

In addition, gold nanomaterials are known for their unique plasmonic resonance features, as well as other promising applications in sensing, imaging, wave guiding, nanoantenna, etc, ${ }^{[6,7]}$ where atomically flat surfaces and single-crystalline features enhance their local plasmonic fields. ${ }^{[7]}$ Owing to their unprecedented planar area, the gold microflakes synthesized in this study may push forward optical nanocircuitry in topdown micro-/nano-fabrication techniques. For example, by nanoskiving layer-by-layer organized microflakes in polymer composites, super long gold nanowire could be prepared (Figure 5A). Other shapes can also be obtained by mechanical processing (e.g., cutting in Figure 5B) and focused ion beam etching (Figures 5C and S10, Supporting Information).

In summary, single-crystal gold microflakes with planar area up to and beyond $10^{4} \mu^{2}$, were fabricated for the first time through a facile, one-step, green approach. By controlling the concentration of $\beta$-lactoglobulin amyloid fibrils, acting as reducing, stabilizing, and nucleating agents, over the concentration of chloroaurate ions, a fine tuning in the dimensions of the gold microflakes could be achieved. The unprecedented macroscopic dimensions of these gold single-crystals offer a number of advantages in the applications involving manipulation of 2D microscopic materials. For example, flexible conductive composite films with hierarchical structures, metallic conductivity, and golden optical surfaces were fabricated through simply casting an aqueous polyurethane suspension containing single-crystal gold microflakes, when homologue films based on single-crystal gold nanosheets yielded insulating materials. These films exhibit a unique stain-controlling conductivity behavior, which paves the way to "on-off" switchers and strain/force/vibration sensing devices. This new type of microflakes can ideally serve in optical nanocircuitry, single-crystal electronics, nanoantenna, sensing, and imaging devices in a broad range of micro-/nano-fabrication techniques.

\section{Supporting Information}

Supporting Information is available from the Wiley Online Library or from the author.

\section{Acknowledgements}

J.Z. and A.S. contributed equally to this work. The Electron Microscopy Centre of the ETH Zurich (EMEZ) is kindly acknowledged for the facility support. We thank the National Natural Science Foundation of China (Grant No. 21474125) and Qingdao Municipal Science and Technology Program (Grant No. 121437-jch) for financial support.

Received: November 8, 2014

Revised: January 16, 2015

Published online: February 5, 2015

[1] C. Li, J. Adamcik, R. Mezzenga, Nat. Nanotechnol. 2012, 7, 421.

[2] A. Walther, I. Bjurhager, J.-M. Malho, J. Ruokolainen, L. Berglund, O. Ikkala, Angew. Chem. Int. Ed. 2010, 49, 6448.

[3] C. Li, A.-K. Born, T. Schweizer, M. Zenobi-Wong, M. Cerruti, R. Mezzenga, Adv. Mater. 2014, 26, 3207.

[4] C. N. R. Rao, H. S. S. Ramakrishna Matte, U. Maitra, Angew. Chem. Int. Ed. 2013, 52, 13162.

[5] L. Brus, Acc. Chem. Res. 2014, 47, 2951.

[6] N. Liu, M. L. Tang, M. Hentschel, H. Giessen, A. P. Alivisatos, Nat. Mater. 2011, 10, 631.

[7] J.-S. Huang, V. Callegari, P. Geisler, C. Brüning, J. Kern, J. C. Prangsma, X. Wu, T. Feichtner, J. Ziegler, P. Weinmann, M. Kamp, A. Forchel, P. Biagioni, U. Sennhauser, B. Hecht, Nat. Commun. 2010, 1, 150

[8] C. Li, S. Bolisetty, R. Mezzenga, Adv. Mater. 2013, 25, 3694.

[9] G. D. Moon, G.-H. Lim, J. H. Song, M. Shin, T. Yu, B. Lim, U. Jeong, Adv. Mater. 2013, 25, 2707

[10] S. Nootchanat, C. Thammacharoen, B. Lohwongwatana, S. Ekgasit, RSC Adv. 2013, 3, 3707.

[11] C. Bouvy, G. A. Baker, H. Yin, S. Dai, Cryst. Growth Des. 2010, 10, 1319.

[12] L. Wang, X. Chen, J. Zhan, Y. Chai, C. Yang, L. Xu, W. Zhuang, B. Jing, J. Phys. Chem. B 2005, 109, 3189.

[13] M. Grzelczak, J. Pérez-Juste, P. Mulvaney, L. M. Liz-Marzán, Chem. Soc. Rev. 2008, 37, 1783.

[14] X. Huang, X. Qi, Y. Huang, S. Li, C. Xue, C. L. Gan, F. Boey, H. Zhang, ACS Nano 2010, 4, 6196.

[15] S. S. Shankar, A. Rai, B. Ankamwar, A. Singh, A. Ahmad, M. Sastry, Nat. Mater. 2004, 3, 482.

[16] J. Jiu, K. Suganuma, M. Nogi, J. Mater. Sci. 2011, 46, 4964.

[17] H. L. Qin, D. Wang, Z. L. Huang, D. M. Wu, Z. C. Zeng, B. Ren, K. Xu, J. Jin, J. Am. Chem. Soc. 2013, 135, 12544.

[18] Z. Li, Z. Liu, J. Zhang, B. Han, J. Du, Y. Gao, T. Jiang, J. Phys. Chem. B 2005, 109, 14445 .

[19] D. Wei, W. Qian, Y. Shi, S. Ding, Y. Xia, Carbohydr. Res. 2007, 342, 2494.

[20] C. A. Goss, D. H. Charych, M. Majda, Anal. Chem. 1991, 63,85 .

[21] J. Hwang, M. A. Dubson, J. Appl. Phys. 1992, 72, 1852.

[22] C. Nogues, M. Wanunu, Surf. Sci. 2004, 573, L383. 
[23] K. Banu, T. Shimura, New J. Chem. 2012, 36, 2112.

[24] T. Kida, Langmuir 2008, 24, 7648.

[25] S. Bolisetty, J. J. Vallooran, J. Adamcik, S. Handschin, F. Gramm, R. Mezzenga, J. Colloid Interface Sci. 2011, 361, 90.

[26] J. Adamcik, J.-M. Jung, J. Flakowski, P. De Los Rios, G. Dietler, R. Mezzenga, Nat. Nanotechnol. 2010, 5, 423.

[27] J.-M. Jung, G. Savin, M. Pouzot, C. Schmitt, R. Mezzenga, Biomacromolecules 2008, 9, 2477.

[28] J.-M. Jung, R. Mezzenga, Langmuir 2010, 26, 504.

[29] J. Wang, L. Lin, Q. Cheng, L. Jiang, Angew. Chem. Int. Ed. 2012, 51, 4676.
[30] D. C. Hyun, M. Park, C. Park, B. Kim, Y. Xia, J. H. Hur, J. M. Kim, J. J. Park, U. Jeong, Adv. Mater. 2011, 23, 2946.

[31] P. Lee, J. Lee, H. Lee, J. Yeo, S. Hong, K. H. Nam, D. Lee, S. S. Lee, S. H. Ko, Adv. Mater. 2012, 24, 3326.

[32] M. Park, J. Im, M. Shin, Y. Min, J. Park, H. Cho, S. Park, M.-B. Shim, S. Jeon, D.-Y. Chung, J. Bae, J. Park, U. Jeong, K. Kim, Nat. Nanotechnol. 2012, 7, 803.

[33] H. Wu, L. Hu, M. W. Rowell, D. Kong, J. J. Cha, J. R. McDonough, J. Zhu, Y. Yang, M. D. McGehee, Y. Cui, Nano Lett. 2010, 10, 4242.

[34] Y. Kim, J. Zhu, B. Yeom, M. Di Prima, X. Su, J.-G. Kim, S. J. Yoo, C. Uher, N. A. Kotov, Nature 2013, 500, 59. 